\title{
Innate lymphoid cells: models of plasticity for immune homeostasis and rapid responsiveness in protection
}

\author{
FF Almeida ${ }^{1,2}$ and GT Belz ${ }^{1,2}$
}

Innate lymphoid cells (ILCs) have stormed onto the immune landscape as "newly discovered" cell types. These tissueresident sentinels are enriched at mucosal surfaces and engage in complex cross talk with elements of the adaptive immune system and microenvironment to orchestrate immune homeostasis. Many parallels exist between innate cells and T cells leading to the initial partitioning of ILCs into rather rigid subsets that reflect their "adaptive-like" effector cytokines profiles. ILCs themselves, however, have unique attributes that are only just beginning to be elucidated. These features result in complementarity with, rather than complete duplication of, functions of the adaptive immune system. Key transcription factors determine the pathway of differentiation of progenitors towards an ILC1, ILC2, or ILC3 subset. Once formed, flexibility in the responses of these subsets to stimuli unexpectedly allows transdifferentation between the different subsets and the acquisition of altered phenotypes and function. This provides a mechanism for rapid innate immune responsiveness. Here, we discuss the models of differentiation for maintenance and activation of tissue-resident ILCs in maintaining immune homeostasis and protection.

\section{INTRODUCTION}

Innate lymphoid cells (ILCs) are a growing family of tissueresident immune cells with important effector functions necessary for immune protection, tissue homeostasis, repair and remodeling. ${ }^{1-4}$ Natural killer (NK) cells, first described in $1975,{ }^{5}$ and lymphoid tissue inducer (LTi) cells, identified nearly a decade later, ${ }^{6}$ are the prototypical members of this family. NK cells mediate early immune responses against viruses and are involved in cytotoxicity while LTi cells are essential for the formation of secondary lymphoid organs during embryogenesis, and for the formation of intestinal lymphoid clusters postnatally. ${ }^{6-11}$ More recently, new members of the ILC family have been described and the family has now been subdivided into three functional groups-group 1 (ILC1), ILC2, and ILC3 (Figure 1). These cells are characterized by their lymphoid morphology, dependency on common gamma chain $(\gamma c)$ signaling, and requirement for the transcription factor inhibitor of DNA-binding 2 (ID2) for their development, ${ }^{12-15}$ and lack recombination-activating gene
(Rag)-dependent rearranged antigen receptors classically found on B and T cells. ${ }^{6}$ As ILCs lack specific adaptive antigen receptors, they rely heavily on both their ability to respond to cytokines through their surface receptors, and to drive interactions between other epithelial and adaptive immune cells through their production of effector cytokines. They have been found to readily respond to a vast array of cytokines including as IL-1, IL-12, IL-7, IL-23, IL-25, IL-33, and thymic stromal lymphopoietin. ${ }^{16,17}$

\section{GROUP 1 ILCs (ILC1s)}

NK cells express the transcription factor T-bet and produce interferon gamma (IFN- $\gamma$ ) (Figure 1) and in many ways mirroring key features of $\mathrm{CD} 8^{+} \mathrm{T}$ cells. They have a critical role in directly killing tumor cells without prior sensitization and participate in a wide variety of immune responses such as viral infections and transplantation. In the mouse, the NK cell family includes several subsets such as circulating splenic and bone marrow NK cells, ${ }^{18} \mathrm{CD} 127^{+}$IL-7-dependent thymic-derived

${ }^{1}$ The Walter and Eliza Hall Institute of Medical Research, Parkville, Victoria, Australia and ${ }^{2}$ Department of Medical Biology, University of Melbourne, Parkville, Victoria, Australia. Correspondence: GT Belz (belz@wehi.edu.au) 


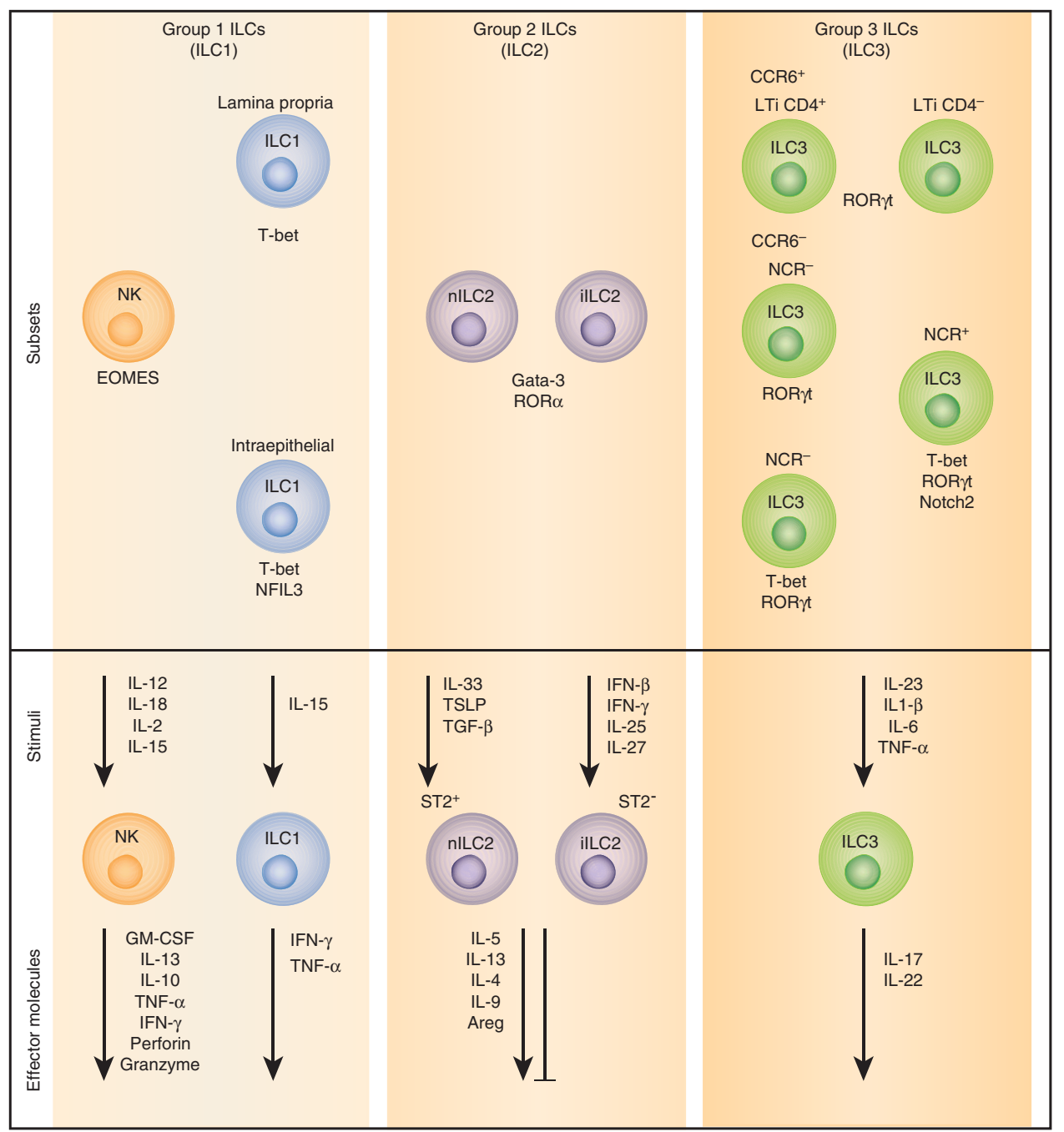

Figure 1 Division of the ILC family at steady-state. Group 1, 2, and 3 ILCs are defined by their differential expression of cell-surface markers, transcription factors, and patterns effector cytokine expression. ILCs can be activated by a diverse range of stimuli and can contribute to immunity, inflammation, and maintenance of tissue homeostasis. Areg, amphiregulin; EOMES, Eomesodermin; Gata-3, GATA Binding Protein 3; GM-CSF, granulocyte macrophage colony-stimulating factor; IFN- $\gamma$, interferon- $\gamma$; IL, interleukin; ILC, innate lymphoid cell; NCR, natural cytotoxicity receptor NKp46; NFIL3, Nuclear factor, interleukin 3 regulated; NK, natural killer; ROR $\alpha$, RAR-related orphan receptor alpha; ROR $\gamma$ t, RAR-related orphan receptor gamma; T-bet, T-box transcription factor; TNF, tumor necrosis factor; TSLP, thymic stromal lymphopoietin.

NK cells, ${ }^{19}$ and a number of subsets of tissue-resident NK cells. $^{20,21}$ Although all NK cell subsets depend on IL-15 and IL-15R $\alpha$ for their development, survival, and maintenance, ${ }^{22}$ they differ in their ability to produce effector cytokines and mediate cytotoxicity. ${ }^{23,24}$

The identification of bona fide ILC1 has been more challenging. Their phenotype has been obscured by the fact that ILC1s, like NK cells, share the expression of receptors such as NK1.1 and the NK cell p46-related protein (NKp46) that have in the past served as an operational definition of NK cells. ${ }^{25}$ This was also found to be the case in humans where ILC1s express CD56, NKG2D, NKp46, and the C-type lectin CD161, markers typically associated with NK cells. ${ }^{26}$ Nevertheless, NK cells can generally be distinguished from ILC1s as they express the transcription factor Eomesodermin (EOMES) which is lacking in ILC1s (Figure 1). In contrast, ILC1s express the integrin CD49a and chemokine receptor CXCR6 (reviewed in Seillet and Belz ${ }^{27}$ ).

\section{GROUP 2 ILCs (ILC2s)}

ILC2s have generally been thought to be the most homogeneous class of ILCs expressing largely conserved markers such as IL-7R $\alpha$, CD25 and Sca-1 in all tissues. ${ }^{3}$ Human ILC2s can be further distinguished by the expression of CD161 and high levels of the prostaglandin D2 receptor CRTH2. ${ }^{28}$

Murine ILC2s can be grouped into two distinct subsets: (i) homeostatic or natural ILC2s (nILC2s) and (ii) inflammatory ILC2s (iILC2s), which are distinguished by their differential expression of the interleukin-1 receptor family member ST2 (Figure 1) ${ }^{29}$ nILC2s $\quad\left(\mathrm{ST} 2{ }^{+}\right.$Thy ${ }^{\text {high }}{ }^{2} \mathrm{KLG} 1^{\text {intermediate }}$ $\mathrm{IL}-17 \mathrm{RB}^{\text {low/- }}$ ) are generally found in the lung and respond predominantly to IL-33, while iILC2s (ST2 ${ }^{-}$Thy $1^{\text {low }}$ KLRG1 $^{\text {high }}$ 
$\mathrm{IL}-17 \mathrm{RB}^{+}$) are located in the lymphoid and parenchymal tissues and are rapidly deployed to other sites in response to IL-25. ${ }^{29}$ In contrast to IL-33, which results in a modest expansion of nILC2, IL-25 elicits a large expansion of ilLC2s in the bone marrow and peripheral blood. Both subsets depend on the transcription factors GATA- $3,{ }^{14}$ ROR $\alpha,{ }^{30,31}$ TCF- $1,{ }^{32,33}$ Notch, ${ }^{32}$ and Bcl11b ${ }^{34,35}$ for their development. ILC2s produce IL-5 and IL-13 in a GATA-3dependent manner, ${ }^{36}$ in addition to IL-4. ${ }^{37}$ ILC2s also produce IL-9 and signaling through the IL-9R is essential for their survival ${ }^{38}$ (Figure 1).

Murine ILC2s can be found in various tissues including adipose tissue-associated lymphoid structures, ${ }^{14}$ gut, ${ }^{39}$ lung, ${ }^{40}$ and the dermis of the skin. ${ }^{41-43}$ They promote a variety of responses including expulsion of parasites, ${ }^{44,45}$ tissue repair and maintenance of lung homeostasis following lung infection, ${ }^{46}$ and can drive airway hyperreactivity following influenza infection. ${ }^{47}$ ILC2s also contribute to the pathogenesis of atopic dermatitis. ${ }^{41-43}$ In the skin, they rapidly respond to the alarmin IL-33, IL-25, and thymic stromal lymphopoietin. ${ }^{41-43}$ In visceral adipose tissue, ILC2s maintain metabolic homeostasis by recruiting eosinophils that are necessary to sustain alternative macrophage activation. ${ }^{48}$ In addition to type 2 cytokines, ILC2s produce amphiregulin (Areg), a member of the epidermal growth factor family, which supports the recovery of epithelial barrier integrity after tissue damage. ${ }^{46}$

\section{GROUP 3 ILCs (ILC3s)}

ILC3s are a highly complex group of innate cells. They are defined molecularly by their dependence on the transcription factor ROR $\gamma t$ but vary in their level of expression of T-bet, cell surface markers, and cytokine production profiles (Figure 1). They are divided into fetal and neonatal LTi cells, which are essential for lymphoid tissue organogenesis, ${ }^{49}$ while adult ILC3 subsets, together with LTi cells, drive tissue repair and immune protection through IL-22 production. ${ }^{50-52}$ In the adult, ILC3s can be found mainly in mucosal tissues such as the small and large intestine, Peyer's patches, and gut-associated lymphoid tissue. ${ }^{8,50,51,53,54}$ Small numbers of ILC3 are also present in the spleen $^{52}$ and lung. ${ }^{55}$

ILC3s are divided into five subsets that can be discriminated on the basis of their expression of CCR6 and their expression of the natural cytotoxicity receptor (NCR), NKp46. They are the $\mathrm{CD}^{+}{ }^{+}$and $\mathrm{CD} 4{ }^{-}$subsets of $\mathrm{CCR} 6{ }^{+} \mathrm{NCR}^{-} \mathrm{ROR} \mathrm{t}^{+} \mathrm{LTi}$ cells, ${ }^{56} \mathrm{NCR}^{-} \mathrm{ROR}_{\mathrm{t}}{ }^{+} \mathrm{T}_{\text {-bet }}{ }^{+}$ILC3s, ${ }^{57} \mathrm{NCR}^{-} \mathrm{ROR} \gamma \mathrm{t}^{+}$ T-bet ${ }^{-}$ILC3s, ${ }^{58}$ and $\mathrm{NCR}^{+} \mathrm{ROR}_{\gamma \mathrm{t}}{ }^{+} \mathrm{T}_{\text {-bet }}{ }^{+}$Notch-dependent ILC3 populations ${ }^{33,57,59,60}$ (Figure 1).

Although all ILC3s express ROR $\gamma$ t, the subsets are complex and appear to arise from divergent progenitors. LTi cells arise from the common innate lymphoid progenitor while non-LTi subsets arise from the later stage, the common helper innate lymphoid progenitor. ${ }^{61}$ Surprisingly, despite the existence of two subsets of adult LTi cells, which are distinguished by their expression of the T-cell co-receptor CD4, few transcriptional differences occur between two populations suggesting that they are not as functionally distinct as first thought. ${ }^{62,63} \mathrm{NCR}^{-}$and $\mathrm{NCR}^{+}$ILC3s appear to have a more linear relationship with
$\mathrm{NCR}^{-}$ILC3s identified as a precursor to $\mathrm{NCR}^{+}$ILC3s, a developmental pathway driven by the transcription factors T-bet and Notch 2 (Figure 2). ${ }^{57,64}$ Nevertheless, despite teasing out a number of details of the development of these cells, the exact relationship between each of these different subsets still remains unclear and will require further dissection.

\section{CLASSIFICATION OF ILC FAMILY MEMBERS-ONE SIZE DOES NOT FIT ALL}

The classification of ILCs into ILC1s, ILC2s, and ILC3s is based on their expression of lineage-defining transcription factors, cytokine production, and functional attributes. Group 1 ILCs depend on the T-box transcription factor T-bet and their canonical production of the cytokines IFN- $\gamma$ and TNF- $\alpha$. Group 2 ILCs depend on Gata-3 and produce IL-5 and IL-13, while group 3 ILCs express the transcription factor nuclear receptor ROR $\gamma \mathrm{t}$ and secrete IL-17 and/or IL-22 (ref. 16) (Figure 1).

Initially, this structure was proposed to reflect the phylogeny of the cells, but the landscape has become more complicated and in an effort to connect the different ILC family members, these groupings are likely to be an oversimplification. For example, ILC1s contain both NK cells and non-NK ILC1s. These two subsets differ in their surface markers and dependence on downstream transcription factors such as nuclear factor interleukin 3 (NFIL3), promyelocytic leukemia zinc finger protein (PLZF), and EOMES, demonstrating that each subset is regulated differently (Figure 1). ${ }^{65}$ ILC3s are also very complex. All ILC3s depend on ROR $\gamma t$ but LTi cells arise from the common innate progenitor, which is upstream of the common helper innate progenitor that gives rise to the other members of the ILC3 family. ${ }^{61}$ Furthermore, ILC3s can transdifferentiate into IFN- $\gamma$-producing ILC1s following the downregulation of ROR $\gamma \mathrm{t}$ and induction of T-bet. ${ }^{61}$ These cells express NK1.1 and are now equipped to produce IL-17 and IFN- $\gamma .{ }^{61}$ Currently, they are known as ex-ROR $\gamma$ t ILC3s or ILC1-like cells. Similarly, ILC2s have recently been shown to be capable of transforming into ILC1-like cells (Figure 2). ${ }^{66}$

Categorizing different ILCs based on individual transcriptional switches and cytokine production appears reasonable, particularly at steady-state, but the remarkable capacity of one ILC type to adopt the phenotype of another ILC family member exposes that such a static model cannot account for the dynamic responsiveness of these cells. Indeed, the phenotype of individual cells likely reflects the integration of multiple complex signals, both intrinsic (transcription factors) and extrinsic (tissue localization and cytokines) that shape their features and functions and drive highly flexible differentiation programs.

\section{INNATE LYMPHOID CELL PLASTICITY AND HOMEOSTASIS}

The positioning of ILCs at mucosal or barrier surfaces implies that they should be able to readily respond to environmental cues. How this would be regulated has been unclear but recent studies begin to shed light on a how a flexible effector program is induced in ILC subsets, thereby allowing them to readily 
adapt to the variety of changing signals, and signaling gradients, they might encounter.

ILC1s are characterized by their capacity to produce IFN $-\gamma$ and dependence on the transcription factor T-bet (Figure 2a). Intriguingly, however, aberrant phenotypes resembling ILC1 (termed "ILC1-like" cells) have now been described which appear to originate from both the ILC2 and ILC3 subsets and also rely on T-bet (Figure 2b). How then is such a transition regulated?

\section{ILC3 $\rightarrow$ ILC1 transition}

The first clues came from the use of ROR $\gamma \mathrm{t}^{\text {gfp }}$ reporter mice, ${ }^{67}$ which have been invaluable in tracing the development of the ILC3 subset. ${ }^{56}$ This resulted in the unexpected discovery that some cells within the ILC1 subset can retain the historical imprint of past ROR $\gamma$ t expression (indicated by green fluorescent protein (GFP) expression), even though they had lost ROR $\gamma t$ and adopted the capacity to produce IFN- $\gamma$, the signature cytokine of ILC1 but not ILC3 ${ }^{25,26,57,68-70}$ In mice, these ILCs were described as "ex-ROR $\gamma \mathrm{t}$ ILC3" cells (Figure 2b). In human ILCs, this transition from ILC3 to ILC1 depended on signals directed through IL-12 that promote T-bet expression. ${ }^{71}$ Surprisingly, this pathway was reversible both in vivo and in vitro with reversion mediated through the action of IL-23, IL-2, IL-1 $\beta$, and retinoic acid. ${ }^{71}$ T-bet itself, by regulation of Notch 2 signaling, also guides the development of NCR ${ }^{+}$ILC3s at steady-state (Figure 2c). ${ }^{57,64}$ However, how the balance between $\mathrm{NCR}^{-}$vs. $\mathrm{NCR}^{+}$ILC3 subsets is maintained is unclear, but we propose that this balance reflects controlled low level inflammatory stimulation that normally occurs in the presence of commensal bacteria "tickling" T-bet. To complicate this picture even further, $\mathrm{NCR}^{+}$ILC3 have recently been shown to differentially depend on Notch2 potentially delineating additional $\mathrm{NCR}^{+}$subtypes. $^{72}$ Thus, whether ILC1-like cells arise equally from all $\mathrm{NCR}^{+}$ILC3s, or alternately only from Notch-dependent or independent differentiation pathways, will need further investigation. The additional step of adopting an ILC1-like phenotype likely reflects a heightened level of inflammation as would occur in infection and thus T-bet acts as a rheostat akin to the T-betdependent inflammatory gradient shown to drive $\mathrm{CD} 8{ }^{+} \mathrm{T}$-cell differentiation. ${ }^{73}$ Thus, ILC3 subsets may also be dynamically regulated by the level of inflammation as a key driver of T-bet levels.

\section{ILC2 $\rightarrow$ ILC1 transition}

It has not been known until more recently whether these hints that some subsets of ILC could alter their phenotype in response to local cues was a more generalized phenomena. ILC2s have now been discovered to be capable of converting to an IFN- $\gamma$ secreting phenotype in both humans and mice. ${ }^{74-76}$ ILC2s have been found to be highly heterogeneous and express a range of receptors that allow them to respond to cytokines such as IL-1 $\beta$, IL-2, IL-12, IL-18, and IL-33. Paralleling the pattern of transition from ILC3 to ILC1, these cytokines can drive amplification of ILC2s and the induction of T-bet and IL-12R $\beta 2$, priming ILC2s for a possible switch towards an

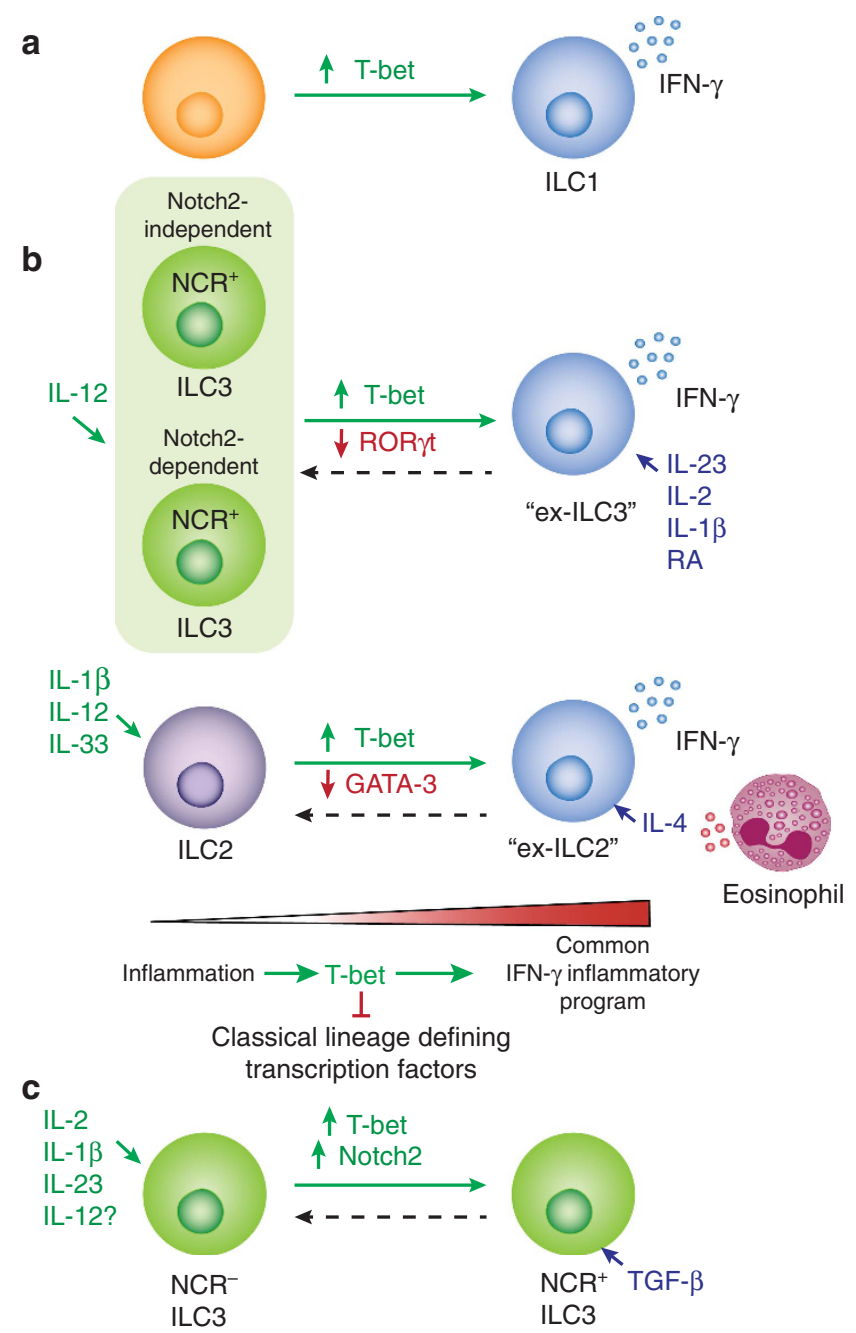

Figure 2 Models of ILC plasticity. (a) ILC1s develop from the lymphoid progenitor following induction of the transcription factor T-bet. (b) ILC3s and ILC2s in peripheral tissues exhibit programs of plasticity in response to cytokine stimulation. Following IL-12 activation, both ILC3s and ILC2s can adopt an ILC1-like phenotype and produce IFN- $\gamma$. IL-12 is essential for the induction of T-bet and augments the inflammatory program in both ILC2s and ILC3s (green arrows). Concurrently, transcription factors such as ROR $\gamma$ t and GATA-3 characteristic of each of these lineages are repressed by T-bet. Graded induction of T-bet but inflammatory stimuli appear to induce a common ILC1 inflammatory program, which is maintained by downregulation of classical lineage defining transcription factors. This pathway can be also reversed (blue arrows) by the action of IL-1 $\beta$, IL-23, IL-2, or RA, or eosinophil-derived IL-4 in the case of ILC3s and ILC2s, respectively. It is unclear whether both Notch 2-dependent and Notch 2independent $\mathrm{NCR}^{+}$ILC3 subsets can both transdifferentiate into ILC1-like cells. (c) Plasticity within the ILC3 subset. NCR ${ }^{-}$ILC3 can differentiate into NCR ${ }^{+}$ILC3 under the influence of T-bet and Notch 2. This pathway appears to be augmented by IL-2, IL-1 $\beta$, IL-23, and IL-12 while TGF- $\beta$ acts as a block inhibiting or limiting NCR ${ }^{+}$ILC3 development and potentially drives reversion of cells. Areg, amphiregulin; EOMES, Eomesodermin; Gata-3, GATA Binding Protein 3; GM-CSF, granulocyte macrophage colony-stimulating factor; IFN- $\gamma$, interferon- $\gamma$; IL, interleukin; ILC, innate lymphoid cell; NCR, natural cytotoxicity receptor NKp46; NFIL3, Nuclear factor, interleukin 3 regulated; NK, natural killer; RA, retinoic acid; ROR $\alpha$, RAR-related orphan receptor alpha; ROR $\gamma \mathrm{t}$, RAR-related orphan receptor gamma; T-bet, T-box transcription factor; TNF, tumor necrosis factor; TSLP, thymic stromal lymphopoietin. 
ILC1-like phenotype. ${ }^{74-76}$ IL-12 was critical specifically to effect genomic modifications of the IFN- $\gamma$ locus to allow the induction of the IFN- $\gamma$ secretion, a feature not seen with other cytokines. ${ }^{75}$ Interestingly, in vitro, this pathway was also reversible. Eosinophil-derived IL-4 was shown to inhibit the IL-12-induced transdifferentiation of ILC2s, thereby favoring the return to an ILC with group 2 function and phenotype. ${ }^{74}$ Precisely how IL-4 regulates these changes in "ex-ILC2" ILC1-like cells will require further characterization (Figure 2b).

This pattern of reversibility of ILC fate has also been observed within the ILC3 subset in response to transforming growth factor- $\beta$ (TGF- $\beta$ ) (Figure 2c) ${ }^{58}$ TGF- $\beta$ is a pleiotropic cytokine secreted by both immune and non-hematopoietic cells and is a potent regulator of immune function. ${ }^{77}$ TGF- $\beta$ signals through the common serine and threonine kinase receptor complex, which has two subunits, TGF- $\beta$ receptor I (TGF- $\beta$ RI) and TGF- $\beta$ RII. The transcripts for both subunits are present on ILCs. ${ }^{78}$ In vivo deletion of TGF- $\beta$ RII resulted in an increase in $\mathrm{NCR}^{+}$ILC3s suggesting that TGF- $\beta$ acts as a block in $\mathrm{NCR}^{+}$ILC3 differentiation. ${ }^{58}$ In line with this, constitutive expression of TGF- $\beta$ RI in TGF $\beta R I^{C A}{ }^{N} \mathrm{cr} 1$-iCre mice significantly impaired the development of the $\mathrm{NCR}^{+}$population. ${ }^{58}$ In vitro analyses mimicked these effects with TGF- $\beta$ signaling able to drive positive signals through Notch, but impaired differentiation when TGF- $\beta$ RI was overexpressed. ${ }^{58}$ Thus, TGF- $\beta$ and Notch have opposing roles in regulating the balance of $\mathrm{NCR}^{-}$and $\mathrm{NCR}^{+}$ILC3s which is likely to be essential in maintaining homeostasis in vivo.

Epithelial-derived TGF- $\beta$ has been shown to have a critical role in the generation of the early immune response by promoting the IL-13-producing ILC2s that drive early allergic immune responses. ${ }^{78}$ The alarmin IL-33 is known to augment the generation of ILC2s. It induces the rapid release of TGF- $\beta$ into the airways to prime ILC2s and enhance their migration. Thus, epithelial-derived TGF- $\beta$ appears to be essential for the accumulation of ILC2s in the lung and acts downstream of IL-33 to initiate type 2 skewed immune response. ${ }^{78}$ Whether it also induces changes in other transcription factors to alter the balance of ILC2 related phenotypes has not yet been investigated, but it is tantalizing to propose that in both ILC2s and ILC3s, TGF- $\beta$ is a key regulator of differentiation and functional attributes in combination with stimuli through other inflammatory mediators. A key question now is precisely which cells are responsible for the production of TGF- $\beta$ in local tissue sites to modulate ILC fate outcomes.

\section{TISSUE-DERIVED CYTOKINES SHAPE ILC FUNCTION IN INFLAMMATION}

Although ILCs are characterized by the distinct cytokine signature they express, their interactions with other cells in the body such as epithelial and immune cells depends largely on extrinsic cytokines originating from epithelium or stromal cells. It is these interactions that drive both positive repair and protection pathways, together with pathogenic pathways that may have negative consequences.
The complex, diverse, and dynamic microbial communities, which colonize the skin and gut pose an immense challenge to the body to maintain immune homeostasis in the face of constantly changing environmental signals. Maintenance of this homeostasis is largely achieved by the action of the IL-22 pathway. In the gut, the direct interaction of surface components of microbes such as flagellin can also induce activation of macrophages and dendritic cells in the lamina propria, activating the IL-22 pathway. This interplay with other cells acts to amplify ILC signaling, particularly ILC3s. Activation of ILC3s by IL-23 subsequently drives IL-22 ref. (79-81) to stimulate epithelial-cell secretion of antimicrobial peptides that inhibit or kill bacteria in the vicinity of the epithelial surface thereby promoting gut mucosal protection. ${ }^{82-84}$ IL-23 has also been shown to be important in the pathogenesis of inflammatory bowel diseases. ${ }^{85}$ Indeed, ILC3s have a role in chronic diseases such as psoriasis, ${ }^{86-91}$ obesity-induced asthma, ${ }^{55}$ and inflammatory bowel diseases, such as Crohn's disease. ${ }^{92}$ Under these inflammatory conditions, myeloid cells (including macrophages and dendritic cells) activated by bacterial stimulation ${ }^{93}$ or alternately, via CD40 signaling, ${ }^{94}$ can induce the production of IL-17 by T helper (Th) 17 cells $^{95-97}$ and ILC3s. ${ }^{98,99} \mathrm{IL}-17 \mathrm{~A}$ is a pro-inflammatory cytokine that has a role in recruitment, activation, and migration of granulocytes, and can also target other cells such as epithelial cells, endothelial cells, fibroblasts, and macrophages to induce cytokines and chemokines ${ }^{100,101}$ to escalate inflammation in affected tissues (Figure 3a).

At steady-state, ILC1s are present at low frequencies in the intestine, while ILC3s are a significantly more prominent subset and contribute to protective innate immune responses, tissue homeostasis, and repair. However, when dysregulated, ILCs can promote inflammation-driven pathology. ${ }^{57,79}$ Indeed, in addition to ILC3s, individuals with Crohn's disease also accumulate ILC1s in their intestinal lamina propria. ${ }^{26}$ These IFN- $\gamma$-producing cells have been shown to further augment the level of inflammation of the affected tissue. ILCs may be altered in diseased tissues by recruitment of subsets from the periphery although it is generally accepted that ILCs are relatively sessile cells that remain in tissues and express limited migration patterns. ${ }^{102}$ Nevertheless, the composition of the ILC populations can change during disease either through the preferential emergence of subsets or perhaps transdifferentiation of one ILC subset into another (see Figure $2 \mathbf{b}$ ). Indeed, bidirectional transdifferentiation between ILC1s and ILC3s in the intestinal lamina propria has recently been reported ${ }^{26,69,71}$ (Figure 3a). It is now clear that ILC3 functional plasticity is controlled by IL-12, IL-23, and IL-1 $\beta$ during inflammation, a feature most clearly demonstrated in vitro. Indeed, distinguishing between the amplification of small subsets of cells and possible transdifferentiation in vivo is challenging. Currently, the exact mechanisms that regulate the switch between the different phenotypes are not clear and will require further investigation. Understanding how this functional plasticity operates in different ILC subsets will be essential to gain insight 
a

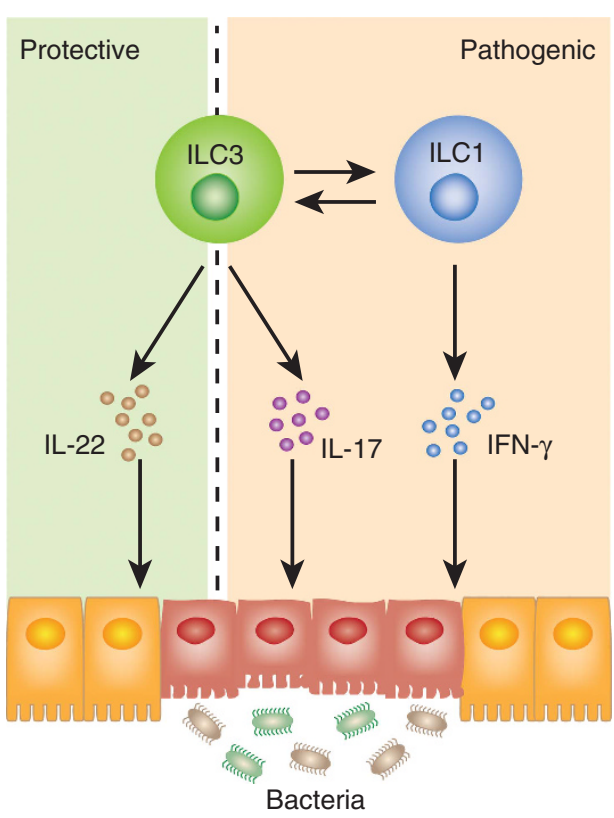

b

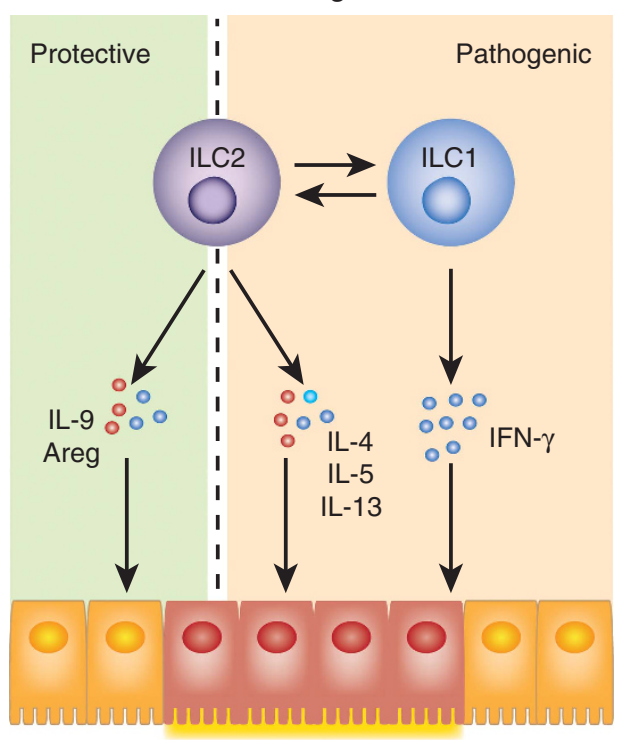

Figure 3 ILC functions in disease. ILCs are amplified in a variety of inflammatory diseases that affect barrier functions and thereby contribute to both immune protection and pathology. (a) In the intestine of Crohn's disease patients, an example of inflammatory bowel disease, ILC3s drive inflammation through their production of IL-17. This contrasts with ILC3-derived-IL-22, which is normally protective and acts to reduce inflammation and induce tissue repair. In addition, transdifferentiation of ILC3s into pathogenic IFN- $\gamma$-producing ILC1-like cells can further amplify the level of inflammation found in the intestinal tissues of patients. (b) In the lung, ILC2s are activated to produce an array of cytokines such as Areg and IL-9, which are involved in repair of the damaged issue, while IL-4, IL-5, and IL-13 lead to airway hyperreactivity and if sustained may lead to chronic inflammation such as occurs in COPD. Following activation with IL-12, ILC2 can transdifferentiate into ILC1 and produce IFN- $\gamma$, which augments inflammation within the epithelium and promotes mucus production. Areg, amphiregulin; COPD, chronic obstructive pulmonary disease; Gata-3, GATA Binding Protein 3; GM-CSF, granulocyte macrophage colony-stimulating factor; IFN- $\gamma$, interferon- $\gamma$; IL, interleukin; ILC, innate lymphoid cell; NCR, natural cytotoxicity receptor NKp46; NFIL3, Nuclear factor, interleukin 3 regulated; NK, natural killer; ROR $\alpha$, RAR-related orphan receptor alpha; ROR $\gamma \mathrm{t}$, RAR-related orphan receptor gamma; T-bet, T-box transcription factor; TNF, tumor necrosis factor; TSLP, thymic stromal lymphopoietin.

to how the balance between protection and pathology are controlled by ILC subsets.

Similar to ILC3s, ILC2s have also been implicated in chronic diseases marked by sustained inflammation such as allergic asthma, ${ }^{103}$ chronic rhinosinusitis, ${ }^{28}$ or atopic dermatitis. ${ }^{43}$ In each of these diseases, activated ILC2s are known to be strong cytokine producers in response of IL-33, IL-25, or TLSP. Cytokines produced by ILC2s can have a dual role. While Areg ${ }^{46}$ and IL- $9^{38}$ have a role in the wound-healing process by promoting tissue remodeling, IL-5, IL-6, IL-4, and IL-13 induce recruitment and activation of other cell types such as mast cells, eosinophils, and macrophages ${ }^{104}$ leading to increased inflammation (Figure 3b). Sustained inflammation can predispose to increased susceptibility to other infections, such as viral, which can result in further enhancement of IL-1 $\beta$ and IL-12 in the tissue. ${ }^{105,106}$ Both cytokines have been implicated in transdifferentiation of ILC2s through an increase of T-bet levels, driving it towards a destructive ILC1 phenotype ${ }^{66}$ (Figure $3 \mathbf{b}$ ). For example, ILC1s have been found to accumulate in the lungs of patients with chronic obstructive pulmonary disease. ${ }^{74}$ Such plasticity in ILC responses would allow rapid reactions to signals, but it may not be ideal when it results in a pathological phenotype. Interestingly, in some chronic diseases, eosinophils accumulate and act as a major source of IL-4. To counterbalance the inflammatory IFN- $\gamma$-producing cells, IL-4 acts to mitigate this pathway allowing ILC1-like cells to convert back to an ILC2 phenotype. ${ }^{74}$ Therefore, IL-12 and IL-4 reciprocally govern ILC2 functional identity and imbalance in this relationship may result in the perpetuation of an inflammatory ILC type. Identification of modulators of ILC functions may be of interest for future therapeutic interventions. For example, specific targeting of the IL-12 pathway would allow modulation of potential IFN- $\gamma$-mediated pathology in the lung thereby ameliorating the progression of emphysema in patients with chronic obstructive pulmonary disease.

In addition to IL-12, type I interferons such as IFN- $\beta$ can have a role in modulating ILC2 function. Expression of type I interferon receptors on ILC2s suggest that they may have a role in suppression of ILC2-mediated inflammatory responses during viral infections. ${ }^{107}$ Indeed, IFN- $\beta$ and IFN- $\gamma$ were discovered to inhibit responses of tissue-resident ILC2s. Similarly, the cytokine, IL-27, a heterodimeric cytokine that belongs to the IL-12 family, has also been shown to antagonize ILC2 proliferation and cytokine production. ${ }^{108}$ Currently, our understanding of how these cytokines affect ILC and their cellular origin is not entirely clear although other immune cells may be an important source. 


\section{PROTECTING THE BODY-COMPLEMENTARITY AND REDUNDANCY}

Teasing apart the contributions of individual ILC subsets has been challenging. Efforts to unravel the attributes of ILCs have mainly been performed in mice that lack the recombinationactivating gene (RAG) in which mature $\mathrm{B}$ and $\mathrm{T}$ cells fail to develop. This approach was predicated on the notion that innate lymphocytes were not believed to require RAG. However, recent evidence indicates that in innate cells, RAG regulates cell-intrinsic hyperresponsiveness and DNA repair mechanisms. ${ }^{109}$ The RAG model has been useful in beginning to understand unique functions ILCs. However, it is now evident that RAG-deficient mice exhibit increased frequencies of innate cells accompanied by enhanced cytokine production in the absence of the adaptive immune compartment and may not represent a completely normal innate compartment. In an additional twist, elimination of adaptive immune cells has masked elucidation of the functions that overlap with adaptive immune cells. ${ }^{63,110}$

It seems intuitive that ILCs would exhibit some level of redundancy with adaptive immune cells. The signature cytokines of ILC1s, ILC2s, and ILC3s mirror those of Th1, Th2, and Th17 helper CD4 ${ }^{+} \mathrm{T}$ cells, while NK cells can be considered as the innate counterparts of cytotoxic CD8 ${ }^{+}$ $\mathrm{T}$ cells. These parallels suggest that a conserved evolutionary program overlays each arm of the immune system. However, it was not until recently that this more complex scenario has been tackled..$^{63,110}$ In earlier experiments, Basu et al. ${ }^{111}$ showed that ILC3 can act during the early stages of Citrobacter rodentium infection but are only essential when the bacterial load is high, while IL-22-producing CD ${ }^{+}$T cells regulate the later stages of infection. In contrast, using an approach in which the $\mathrm{NCR}^{+}$ ILC3s can be selectively deleted in an immunocompetent host, or their production of IL-22 abolished, it was shown that they were not essential to control of colonic infection. ${ }^{63}$ A similar effect was observed in a separate study by Colonna et al. ${ }^{110}$ thus highlighting the evolution of multiple checkpoints spanning both arms of the immune system that ensure immune protection. Nevertheless, two key findings emerged from these studies. First, $\mathrm{NCR}^{+}$ILC3s were shown to have a unique role in producing GM-CSF, which was important to drive the recruitment of inflammatory monocytes to sites of infection. ${ }^{110}$ Second, $\mathrm{NCR}^{+}$ILC3s were also shown to have a novel role in maintaining the homeostasis of the cecum and work coordinately with $\mathrm{CD} 4{ }^{+} \mathrm{T}$ cells to protect against bacterial infection. $^{63}$

Crossover between adaptive and innate attributes has also been detected in ILC that coexpress a number of receptors normally associated with adaptive immune cells. Murine LTi-like ILC3s and ILC3s express major histocompatibility complex class II molecules and can present antigen to $\mathrm{CD} 4{ }^{+}$ T cells. ${ }^{112,113}$ Although major histocompatibility complex class II expression on ILC3s can induce immune activation and proliferation as normally accompanies antigen presentation, ${ }^{112}$ this expression in LTi-like ILC3s can also direct tolerization of $\mathrm{T}$ cells to antigens derived from commensal flora. ${ }^{113}$ In the absence of such antigen presentation, mice developed intestinal inflammation, enhanced T-cell proliferation, and increased serum antibody titers against commensal bacteria. ${ }^{113}$ ILC2s have also been discovered to express major histocompatibility complex class II and the costimulatory molecule ICOS (inducible T-cell costimulator), suggesting they may also be involved in presenting antigens in the context of Th2 responses. $^{30,40,114}$ Although the mechanisms that underpin this pathway are yet to be worked out, it exposes an unexpected complementary relationship between innate and adaptive immunity.

\section{CONCLUSIONS AND FUTURE DIRECTIONS}

Mucosal surfaces are the battleground for the immune system, pathogens, and dietary and environmental antigens. Although the role of the adaptive immune system has received considerable attention, exciting developments in understanding the innate immune cells that contribute to the landscape have added an entirely new dimension to how we might view cross talk between the innate and adaptive arms of the system.

Elucidation of the developmental pathways of ILCs has been complex and controversial. Still, much needs to be worked out about the key transcriptional regulators that guide ILC differentiation. The new evidence that transdifferentiation can occur among the different ILC subsets opens new questions for the field. Although it is likely that many cells are generated from local expansion and differentiation, the possibility that transdifferentiation may also occur and to what extent is yet to be resolved. Further investigation will be required to elucidate how and when ILC plasticity emerges in vivo. Given the apparent limited peripheral ILC progenitor pool in tissues and their apparent restricted migration, a flexible differentiation program would allow ILCs to respond rapidly to the immune challenges. Currently, the field has viewed that a reduction in a particular ILC subset reflects absolute depletion. ${ }^{115,116}$ However, it is likely that in some circumstances at least, it is the relative ratio of ILC subsets that is critical in driving immune defense or pathology. Undeniably, much less is understood about the molecular wiring that regulates ILC activation and responsiveness at steady-state or during an infectious challenge. This is likely to be a key area for future investigation to understand how weak vs. strong commensal and pathogenic microorganisms shape the balance of ILC subsets and their fate outcome. Indeed, the features that characterize activation or suppression of ILCs have received little attention, perhaps because they challenge us to consider new models of immune cell regulation. A more complete understanding of the receptors and behavior of ILCs should lead to new approaches to immunotherapies where selective manipulation of ILC subtypes can be used to ameliorate or block disease or promote tissue repair. We also do not understand how unique features of ILCs, such as radio resistance, ${ }^{102}$ might impact immunity and discriminate between the redundant and complementary features of innate and adaptive responses. We have become so accustomed to partitioning different parts of the immune response into discrete units to facilitate our understanding of 
the different elements. Now, it will be necessary to understand how each of these pieces of the puzzle fit together and how even subtle signals can change the outcome. It is very clear that the system is not linear, but represents a highly integrated and regulated switchboard that cannot be fully understood by looking at everything independently.

\section{ACKNOWLEDGMENTS}

We thank C. Seillet for critical reading of the manuscript and discussions. G.T.B is supported by an Australian Research Council Future Fellowship. This work was supported by grants of the National Health and Medical Research Council (NHMRC) of Australia, Victorian State Government Operational Infrastructure Support and Australian Government NHMRC IRIIS.

\section{DISCLOSURE}

The authors declared no conflict of interest.

c) 2016 Society for Mucosal Immunology

\section{REFERENCES}

1. Cortez, V.S., Robinette, M.L. \& Colonna, M. Innate lymphoid cells: new insights into function and development. Curr. Opin. Immuno/ 32C, 71-77 (2015).

2. McKenzie, A.N.J., Spits, H. \& Eberl, G. Innate lymphoid cells in inflammation and immunity. Immunity 41, 366-374 (2014).

3. Diefenbach, A., Colonna, M. \& Koyasu, S. Development, differentiation, and diversity of innate lymphoid cells. Immunity 41, 354-365 (2014).

4. Cella, M., Miller, H. \& Song, C. Beyond NK cells: The expanding universe of innate lymphoid cells. Front. Immunol 5, 1-11 (2014).

5. Kiessling, R., Klein, E., Pross, H. \& Wigzell, H. "Natural" killer cells in the mouse. II. Cytotoxic cells with specificity for mouse Moloney leukemia cells. Characteristics of the killer cell. Eur. J. Immunol. 5, 117-121 (1975).

6. Mebius, R.E., Rennert, P. \& Weissman, I.L. Developing lymph nodes collect CD4 + CD3- LTbeta + cells that can differentiate to APC, NK cells, and follicular cells but not T or B cells. Immunity 7, 493-504 (1997).

7. van de Pavert, S.A. et al. Maternal retinoids control type 3 innate lymphoid cells and set the offspring immunity. Nature 508, 123-127 (2014).

8. Cupedo, T. et al. Human fetal lymphoid tissue-inducer cells are interleukin 17-producing precursors to RORC + CD127 + natural killer-like cells. Nat. Immunol. 10, 66-74 (2009).

9. Hamada, H. et al. Identification of multiple isolated lymphoid follicles on the antimesenteric wall of the mouse small intestine. J. Immunol. 168, 57-64 (2002)

10. Bouskra, D. et al. Lymphoid tissue genesis induced by commensals through NOD1 regulates intestinal homeostasis. Nature 456, 507-510 (2008).

11. Kanamori, Y. et al. Identification of novel lymphoid tissues in murine intestinal mucosa where clusters of c-kit $+\mathrm{IL}-7 \mathrm{R}+$ Thy $1+$ lymphohemopoietic progenitors develop. J. Exp. Med. 184, 1449-1459 (1996).

12. Boos, M.D., Yokota, Y., Eberl, G. \& Kee, B.L. Mature natural killer cell and lymphoid tissue-inducing cell development requires Id2-mediated suppression of E protein activity. J. Exp. Med. 204, 1119-1130 (2007).

13. Yokota, Y. et al. Development of peripheral lymphoid organs and natural killer cells depends on the helix-loop-helix inhibitor Id2. Nature 397, 702-706 (1999).

14. Moro, $\mathrm{K}$. et al. Innate production of $\mathrm{T}(\mathrm{H}) 2$ cytokines by adipose tissueassociated c-Kit $(+)$ Sca-1(+) lymphoid cells. Nature 463, 540-544 (2010).

15. Satoh-Takayama, N. et al. IL-7 and IL-15 independently program the differentiation of intestinal CD3-NKp46 + cell subsets from Id2-dependent precursors. J. Exp. Med. 207, 273-280 (2010).

16. Spits, H. et al. Innate lymphoid cells-a proposal for uniform nomenclature. Nat. Rev. Immunol. 13, 145-149 (2013).

17. Seillet, C., Belz, G.T. \& Mielke, L.A. Complexity of cytokine network regulation of innate lymphoid cells in protective immunity. Cytokine 70 , 1-10 (2014).
18. Hayakawa, Y. \& Smyth, M.J. CD27 dissects mature NK cells into two subsets with distinct responsiveness and migratory capacity. J. Immunol. 176, 1517-1524 (2006).

19. Vosshenrich, C.A.J. et al. A thymic pathway of mouse natural killer cell development characterized by expression of GATA-3 and CD127. Nat. Immunol. 7, 1217-1224 (2006).

20. Yu, J., Freud, A.G. \& Caligiur, M.A. Location and cellular stages of natural killer cell development. Trends Immunol. 34, 573-582 (2013).

21. Yokoyama, W.A.M., Sojka, D.O.K., Peng, H. \& Tian, Z. Tissue-resident natural killer cells. Cold Spring Harb. Symp. Quant. Biol 78, 149-156 (2013). LXXVIII.

22. Cheng, M. et al. Distinct and overlapping patterns of cytokine regulation of thymic and bone marrow-derived NK cell development. J. Immunol. 182, 1460-1468 (2009).

23. Daussy, C. et al. T-bet and Eomes instruct the development of two distinct natural killer cell lineages in the liver and in the bone marrow. J. Exp. Med. 211, 563-577 (2014).

24. Tessmer, M.S., Reilly, E.C. \& Brossay, L. Salivary gland NK cells are phenotypically and functionally unique. PLoS Pathog. 7, e1001254 (2011).

25. Fuchs, A. et al. Intraepithelial type 1 innate lymphoid cells are a unique subset of IL-12- and IL-15-responsive IFN- $\gamma$-producing cells. Immunity 38, 769-781 (2013).

26. Bernink, J.H. et al. Human type 1 innate lymphoid cells accumulate in inflamed mucosal tissues. Nat. Immunol. 14, 221-229 (2013).

27. Seillet, C. \& Belz, G.T. Differentiation and diversity of subsets in group 1 innate lymphoid cells. Int. Immunol. 28, 3-11 (2016).

28. Mjösberg, J.M. et al. Human IL-25- and IL-33-responsive type 2 innate lymphoid cells are defined by expression of CRTH2 and CD161. Nat. Immunol. 12, 1055-1062 (2011).

29. Guo, L., Huang, Y., Chen, X., Hu-Li, J., Urban, J.F. Jr. \& Paul, W.E. Innate immunological function of TH2 cells in vivo. Nat. Immunol. 16, 1051-1059 (2015).

30. Halim, T.Y.F., MacLaren, A., Romanish, M.T., Gold, M.J., McNagny, K.M. \& Takei, F. Retinoic-acid-receptor-related orphan nuclear receptor alpha is required for natural helper cell development and allergic inflammation. Immunity 37, 463-474 (2012).

31. Wong, S.H. et al. Transcription factor RORalpha is critical for nuocyte development. Nat. Immunol 13, 229-236 (2012).

32. Yang, Q. et al. T cell factor 1 is required for group 2 innate lymphoid cell generation. Immunity 38, 694-704 (2013).

33. Mielke, L.A. et al. TCF-1 controls ILC2 and NKp46 + RORgammat + innate lymphocyte differentiation and protection in intestinal inflammation. J. Immunol. 191, 4383-4391 (2013).

34. Yu, Y. et al. The transcription factor Bcl11b is specifically expressed in group 2 innate lymphoid cells and is essential for their development. J. Exp. Med. 212, 865-874 (2015).

35. Walker, J.A. et al. Bcl11b is essential for group 2 innate lymphoid cell development. J. Exp. Med. 212, 875-882 (2015).

36. Yagi, R. et al. The transcription factor GATA3 is critical for the development of all IL-7R $\alpha$-expressing innate lymphoid cells. Immunity 40, 378-388 (2014).

37. Drake, L.Y., lijima, K. \& Kita, H. Group 2 innate lymphoid cells and CD4 + Tcells cooperate to mediate type 2 immune response in mice. Allergy 69 , 1300-1307 (2014).

38. Turner, J.-E. et al. IL-9-mediated survival of type 2 innate lymphoid cells promotes damage control in helminth-induced lung inflammation. J. Exp. Med. 210, 2951-2965 (2013).

39. Neill, D.R. et al. Nuocytes represent a new innate effector leucocyte that mediates type-2 immunity. Nature 464, 1367-1370 (2010).

40. Halim, T.Y., Krauss, R.H., Sun, A.C. \& Takei, F. Lung natural helper cells are a critical source of Th2 cell-type cytokines in protease allergeninduced airway inflammation. Immunity 36, 451-463 (2012).

41. Roediger, B. et al. Cutaneous immunosurveillance and regulation of inflammation by group 2 innate lymphoid cells. Nat. Immunol. 14, 564-573 (2013).

42. Imai, Y. et al. Skin-specific expression of IL-33 activates group 2 innate lymphoid cells and elicits atopic dermatitis-like inflammation in mice. Proc. Natl Acad. Sci. USA 110, 13921-13926 (2013).

43. Salimi, M. et al. A role for IL-25 and IL-33-driven type-2 innate lymphoid cells in atopic dermatitis. J. Exp. Med. 210, 2939-2950 (2013). 
44. Fallon, P.G. et al. Identification of an interleukin (IL)-25-dependent cell population that provides IL-4, IL-5, and IL-13 at the onset of helminth expulsion. J. Exp. Med. 203, 1105-1116 (2006).

45. Walker, J.A. \& McKenzie, A.N.J. Development and function of group 2 innate lymphoid cells. Curr. Opin. Immunol. 25, 148-155 (2013).

46. Monticelli, L.A. et al. Innate lymphoid cells promote lung-tissue homeostasis after infection with influenza virus. Nat. Immunol. 12, 1045-1054 (2011).

47. Chang, Y.-.J et al. Innate lymphoid cells mediate influenza-induced airway hyper-reactivity independently of adaptive immunity. Nat. Immunol. 12, 631-638 (2011).

48. Lee, M.W. et al. Activated type 2 innate lymphoid cells regulate beige fat biogenesis. Cell 160, 74-87 (2015).

49. van de Pavert, S.A. \& Mebius, R.E. New insights into the development of lymphoid tissues. Nat. Rev. Immunol. 10, 664-674 (2010).

50. Luci, C. et al. Influence of the transcription factor RORgammat on the development of NKp46 + cell populations in gut and skin. Nat. Immunol. 10, 75-82 (2009)

51. Sanos, S.L. et al. RORgammat and commensal microflora are required for the differentiation of mucosal interleukin 22-producing NKp46 + cells. Nat. Immunol. 10, 83-91 (2009).

52. Takatori, H. et al. Lymphoid tissue inducer-like cells are an innate source of IL-17 and IL-22. J. Exp. Med. 206, 35-41 (2009).

53. Cella, M. et al. A human natural killer cell subset provides an innate source of IL-22 for mucosal immunity. Nature 457, 722-725 (2009).

54. Satoh-Takayama, N. et al. Microbial flora drives interleukin 22 production in intestinal NKp46 + cells that provide innate mucosal immune defense. Immunity 29, 958-970 (2008).

55. Kim, H.Y. et al. Interleukin-17-producing innate lymphoid cells and the NLRP3 inflammasome facilitate obesity-associated airway hyperreactivity. Nat. Med. 20, 54-61 (2014).

56. Sawa, S. et al. Lineage relationship analysis of RORgammat + innate lymphoid cells. Science 330, 665-669 (2010).

57. Klose, C.S. et al. A T-bet gradient controls the fate and function of CCR6-RORgammat + innate lymphoid cells. Nature 494, 261-265 (2013).

58. Viant, C. et al. Transforming growth factor-beta and Notch ligands act as opposing environmental cues in regulating the plasticity of type 3 innate lymphoid cells. Sci. Signal. 9, ra46 (2016).

59. Lee, J.S. et al. AHR drives the development of gut ILC22 cells and postnatal lymphoid tissues via pathways dependent on and independent of Notch. Nat. Immunol. 13, 144-151 (2011).

60. Sciumé, G. et al. Distinct requirements for T-bet in gut innate lymphoid cells. J. Exp. Med. 209, 2331-2338 (2012).

61. Ishizuka, I.E. et al. Single-cell analysis defines the divergence between the innate lymphoid cell lineage and lymphoid tissue-inducer cell lineage. Nat. Immunol. 17, 269-276 (2016).

62. Robinette, M.L. et al. Transcriptional programs define molecular characteristics of innate lymphoid cell classes and subsets. Nat. Immunol. 16, 306-317 (2015).

63. Rankin, L.C. et al. Complementarity and redundancy of IL-22-producing innate lymphoid cells. Nat. Immunol. 17, 179-186 (2016).

64. Rankin, L.C. et al. The transcription factor T-bet is essential for the development of NKp46(+) innate lymphocytes via the Notch pathway. Nat. Immunol. 14, 389-395 (2013).

65. Serafini, N., Vosshenrich, C.A. \& Di Santo, J.P. Transcriptional regulation of innate lymphoid cell fate. Nat. Rev. Immunol. 15, 415-428 (2015).

66. Lim, A.I. et al. IL-12 drives functional plasticity of human group 2 innate lymphoid cells. J. Exp. Med. 213, 569-583 (2016).

67. Eberl, G., Marmon, S., Sunshine, M.J., Rennert, P.D., Choi, Y. \& Littman, D.R. An essential function for the nuclear receptor RORgamma(t) in the generation of fetal lymphoid tissue inducer cells. Nat. Immunol. 5, 64-73 (2004).

68. Klose, C.S.N. et al. Differentiation of type 1 ILCs from a common progenitor to all helper-like innate lymphoid cell lineages. Cell 157, 340-356 (2014).

69. Vonarbourg, C. et al. Regulated expression of nuclear receptor ROR $\gamma t$ confers distinct functional fates to NK cell receptor-expressing ROR $\gamma \mathrm{t}(+)$ innate lymphocytes. Immunity 33, 736-751 (2010).
70. Cella, M., Otero, K. \& Colonna, M. Expansion of human NK-22 cells with IL-7, IL-2, and IL-1beta reveals intrinsic functional plasticity. Proc. Natl. Acad. Sci. USA 107, 10961-10966 (2010).

71. Bernink, J.H. et al. Interleukin-12 and -23 control plasticity of $\operatorname{cd} 127(+)$ group 1 and group 3 innate lymphoid cells in the intestinal lamina propria. Immunity 43, 146-160 (2015).

72. Chea, S. et al. Notch signaling in group 3 innate lymphoid cells modulates their plasticity. Sci. Signal. 9, ra45 (2016).

73. Joshi, N.S. et al. Inflammation directs memory precursor and short-lived effector CD8(+) $\mathrm{T}$ cell fates via the graded expression of T-bet transcription factor. Immunity 27, 281-295 (2007).

74. Bal, S.M. et al. IL-1beta, IL-4 and IL-12 control the fate of group 2 innate lymphoid cells in human airway inflammation in the lungs. Nat. Immunol. 17, 636-645 (2016).

75. Ohne, Y. et al. IL-1 is a critical regulator of group 2 innate lymphoid cell function and plasticity. Nat. Immunol. 17, 646-655 (2016).

76. Silver, J.S. et al. Inflammatory triggers associated with exacerbations of COPD orchestrate plasticity of group 2 innate lymphoid cells in the lungs. Nat. Immunol. 17, 626-635 (2016).

77. Travis, M.A. \& Sheppard, D. TGF-beta activation and function in immunity. Annu. Rev. Immunol. 32, 51-82 (2014).

78. Denney, L. et al. Pulmonary epithelial cell-derived cytokine TGF-beta1 is a critical cofactor for enhanced innate lymphoid cell function. Immunity 43 , 945-958 (2015).

79. Buonocore, S. et al. Innate lymphoid cells drive interleukin-23-dependent innate intestinal pathology. Nature 464, 1371-1375 (2010).

80. Colonna, M. Interleukin-22-producing natural killer cells and lymphoid tissue inducer-like cells in mucosal immunity. Immunity 31, 15-23 (2009).

81. Sonnenberg, G.F., Fouser, L.A. \& Artis, D. Border patrol: regulation of immunity, inflammation and tissue homeostasis at barrier surfaces by IL-22. Nat. Immunol. 12, 383-390 (2011).

82. Kinnebrew, M.A. et al. Interleukin 23 production by intestinal CD103(+) $\mathrm{CD11b}(+)$ dendritic cells in response to bacterial flagellin enhances mucosal innate immune defense. Immunity 36, 276-287 (2012).

83. Kinnebrew, M.A., Ubeda, C., Zenewicz, L.A., Smith, N., Flavell, R.A. \& Pamer, E.G. Bacterial flagellin stimulates Toll-like receptor 5-dependent defense against vancomycin-resistant Enterococcus infection. J. Infect. Dis. 201, 534-543 (2010).

84. Van Maele, L. et al. TLR5 signaling stimulates the innate production of $\mathrm{IL}-17$ and IL-22 by CD3(neg)CD127 + immune cells in spleen and mucosa. J. Immunol. 185, 1177-1185 (2010).

85. McGovern, D. \& Powrie, F. The IL23 axis plays a key role in the pathogenesis of IBD. Gut 56, 1333-1336 (2007).

86. Piskin, G., Sylva-Steenland, R.M., Bos, J.D. \& Teunissen, M.B. In vitro qand in situ expression of IL-23 by keratinocytes in healthy skin and psoriasis lesions: enhanced expression in psoriatic skin. J. Immunol. 176, 1908-1915 (2006).

87. Lee, E. et al. Increased expression of interleukin 23 p19 and p40 in lesional skin of patients with psoriasis vulgaris. J. Exp. Med. 199, 125-130 (2004).

88. Leonardi, C. et al. Anti-interleukin-17 monoclonal antibody ixekizumab in chronic plaque psoriasis. N. Engl. J. Med. 366, 1190-1199 (2012).

89. Papp, K.A. et al. Brodalumab, an anti-interleukin-17-receptor antibody for psoriasis. N. Engl. J. Med. 366, 1181-1189 (2012).

90. Tonel, G. et al. Cutting edge: A critical functional role for IL-23 in psoriasis. J. Immunol. 185, 5688-5691 (2010).

91. Zaba, L.C. etal. Amelioration of epidermal hyperplasia by TNF inhibition is associated with reduced Th17 responses. J. Exp. Med. 204, 3183-3194 (2007).

92. Geremia, A. et al. IL-23-responsive innate lymphoid cells are increased in inflammatory bowel disease. J. Exp. Med. 208, 1127-1133 (2011).

93. Becker, C. et al. Constitutive p40 promoter activation and IL-23 production in the terminal ileum mediated by dendritic cells. J. Clin. Invest. 112, 693-706 (2003).

94. Uhlig, H.H. et al. Differential activity of IL-12 and IL-23 in mucosal and systemic innate immune pathology. Immunity 25, 309-318 (2006).

95. Langrish, C.L. et al. IL-23 drives a pathogenic T cell population that induces autoimmune inflammation. J. Exp. Med. 201, 233-240 (2005).

96. Aggarwal, S., Ghilardi, N., Xie, M.H., de Sauvage, F.J. \& Gurney, A.L. Interleukin-23 promotes a distinct CD4 $\mathrm{T}$ cell activation state 
characterized by the production of interleukin-17. J. Biol. Chem. 278, 1910-1914 (2003).

97. Harrington, L.E. et al. Interleukin 17-producing CD4 + effector T cells develop via a lineage distinct from the T helper type 1 and 2 lineages. Nat. Immunol. 6, 1123-1132 (2005).

98. Wolk, K., Kunz, S., Witte, E., Friedrich, M., Asadullah, K. \& Sabat, R. IL-22 increases the innate immunity of tissues. Immunity 21, 241-254 (2004).

99. Satoh-Takayama, N. et al. The chemokine receptor CXCR6 controls the functional topography of interleukin-22 producing intestinal innate lymphoid cells. Immunity 41, 776-788 (2014).

100. Korn, T., Bettelli, E., Oukka, M. \& Kuchroo, V.K. IL-17 and Th17 Cells. Annu. Rev. Immunol. 27, 485-517 (2009).

101. Iwakura, Y., Ishigame, H., Saijo, S. \& Nakae, S. Functional specialization of interleukin-17 family members. Immunity 34, 149-162 (2011).

102. Gasteiger, G., Fan, X., Dikiy, S., Lee, S.Y. \& Rudensky, A.Y. Tissue residency of innate lymphoid cells in lymphoid and nonlymphoid organs. Science 350, 981-985 (2015).

103. Nagakumar, P., Denney, L., Fleming, L., Bush, A., Lloyd, C.M. \& Saglani, S. Type 2 innate lymphoid cells in induced sputum from children with severe asthma. J. Allergy Clin. Immunol. 137, 624-626 e626 (2016).

104. Nussbaum, J.C. et al. Type 2 innate lymphoid cells control eosinophil homeostasis. Nature 502, 245-248 (2013).

105. MacDonald, M., Korman, T., King, P., Hamza, K. \& Bardin, P. Exacerbation phenotyping in chronic obstructive pulmonary disease. Respirology 18, 1280-1281 (2013).

106. Mohan, A. et al. Prevalence of viral infection detected by PCR and RT-PCR in patients with acute exacerbation of COPD: a systematic review. Respirology 15, 536-542 (2010).
107. Duerr, C.U. et al. Type I interferon restricts type 2 immunopathology through the regulation of group 2 innate lymphoid cells. Nat. Immunol. 17, 65-75 (2016).

108. Moro, K. et al. Interferon and IL-27 antagonize the function of group 2 innate lymphoid cells and type 2 innate immune responses. Nat. Immunol. 17, 76-86 (2016).

109. Karo, J.M., Schatz, D.G. \& Sun, J.C. The RAG recombinase dictates functional heterogeneity and cellular fitness in natural killer cells. Cell 159, 94-107 (2014).

110. Song, C. et al. Unique and redundant functions of NKp46 + ILC3s in models of intestinal inflammation. J. Exp. Med. 212, 1869-1882 (2015).

111. Basu, R. etal. Th22 cells are an important source of IL-22 for host protection against enteropathogenic bacteria. Immunity 37, 1061-1075 (2012).

112. von Burg, N. et al. Activated group 3 innate lymphoid cells promote T-cell-mediated immune responses. Proc. Natl. Acad. Sci. USA 111, 12835-12840 (2014).

113. Hepworth, M.R. et al. Innate lymphoid cells regulate CD4 + T-cell responses to intestinal commensal bacteria. Nature 498, 113-117 (2013).

114. Bartemes, K.R., lijima, K., Kobayashi, T., Kephart, G.M., McKenzie, A.N. \& Kita, H. IL-33-responsive lineage- CD25 + CD44(hi) lymphoid cells mediate innate type 2 immunity and allergic inflammation in the lungs. J. Immunol. 188, 1503-1513 (2012).

115. Zhang, Z. et al. Plasmacytoid dendritic cells promote HIV-1-induced group 3 innate lymphoid cell depletion. J. Clin. Invest. 125, 3692-3703 (2015).

116. Kloverpris, H.N. et al. Innate lymphoid cells are depleted irreversibly during acute HIV-1 infection in the absence of viral suppression. Immunity 44, 391-405 (2016). 